\title{
Color agreement between nanofluorapatite ceramic discs associated with try-in pastes and with resin cements
}

Paulo Rigoni(a)

Flávia Lucisano Botelho do Amaral(b)

Fabiana Mantovani Gomes

França $\mathbf{a}^{(b)}$

Roberta Tarkany Basting ${ }^{(b)}$

(a) Department of Prosthodontics, School of Dentistry, São Leopoldo Mandic Dental Research Institute, Campinas, SP, Brazil.

(b) Department of Restorative Dentistry, School of Dentistry, São Leopoldo Mandic Dental Research Institute, Campinas, SP, Brazil.
Declaration of Interests: The authors certify that they have no commercial or associative interest that represents a conflict of interest in connection with the manuscript.

Corresponding Author: Roberta Tarkany Basting

E-mail: rbasting@yahoo.com

Submitted: May 28, 2012

Accepted for publication: Sep 17, 2012

Last revision: Oct 01, 2012

\begin{abstract}
The aim of this study was to evaluate the in vitro color agreement between nanofluorapatite ceramic discs (e.max Ceram/Ivoclar Vivadent/A2) associated with try-in pastes and those bonded with resin cements (Vitique/DMG/try-in shade A2 $1 \frac{1}{2}$ and cement shade A2 $1 \frac{1}{2}$, Variolink II/Ivoclar Vivadent/try-in shade A1 and cement shade A1, and Choice 2/Bisco/try-in shade A2 and cement shade A2), and to evaluate the shade stability of the discs bonded with resin cements. The shades of composite resin discs (Lliss/FGM/A2) and nanofluorapatite ceramic discs with try-in pastes or cements were evaluated according to the Vita Classical shade guide by a digital spectrophotometer (Micro EspectroShade, MHT) immediately after placing the try-in pastes or resin cements between composite resin discs and ceramic discs. Other evaluations were performed at 2, 5, and 6 day intervals after cementation with the resin cements. All ceramic discs that received try-in pastes presented an A2 shade. There was no statistical difference in the shade of the ceramic specimens fixed with different cements at the different intervals, as evaluated by the Friedman test $(\mathrm{p}>0.05)$. Two try-in pastes presented shade compatibility with those recommended by the manufacturers. There was no similarity of shades between the ceramic discs with try-in pastes and those with the respective resin cements. Shade stability was observed in ceramic discs with resin cements within the intervals evaluated.
\end{abstract}

Descriptors: Color Perception; Ceramics; Resin Cements.

\section{Introduction}

The importance of shade selection for an indirect restoration is connected to the perception of chromatic hues involved in the dental structure. ${ }^{1-3}$ Any mistake in shade selection and/or communication with the laboratory technician may compromise the esthetics of the rehabilitation. This is a common failure because shade selection is based on subjective methods and on existent color scales or photographs. ${ }^{4}$

Among dental ceramics, the nanofluorapatite vitreous type used for the esthetic lining of ceramic infrastructures in laminate veneers has shown satisfactory esthetic and functional results, ${ }^{5,6}$ with an estimated survival probability of $93.5 \%$ over 10 years. ${ }^{6}$ The optical properties of apatite crystals are related to the nanometric size and type of nanofluor- 
apatite crystals with micrometer length scales. Since nanofluorapatite ceramics contain different concentrations of apatite crystals, they are responsible for greater control of the translucence, fluorescence, and opalescence ${ }^{6,7}$ that allow natural light distribution for a balanced relationship between hue, value, and chrome, thus making nanofluorapatite similar to natural teeth. ${ }^{8}$ However, in very thin restorations, such as laminate veneers, the cement and substrate shades, as well as the thickness of the resin cement layer, may influence the esthetics of the restoration. One method of selecting the cement shade is by using try-in pastes prior to cementation procedures. ${ }^{9-11}$

Try-in pastes are composed of water-soluble pigmented glycerin, and are made with a consistency and shade similar to those of resin cements, in order to simulate the chromatic effect promoted by the completely polymerized resin cement after light activation. ${ }^{10}$ Try-in pastes are handled in a way similar to the handling of resin cements, and have to be removed from the preparation after shade selection, because the glycerin residues may compromise the bonding between resin cement and tooth. ${ }^{11,12}$

Some studies have evaluated the shade agreement between the try-in systems and their respective cements, and have found no significant differences. ${ }^{13,14}$ Only Alghazali et al. ${ }^{15}$ evaluated shade agreement after dental ceramic cementation. Therefore, not only must there be agreement between try-in pastes and resin cements, but cement shade stability after light activation must also be considered, insofar as the shade may change over time, thus compromising the esthetics. ${ }^{16-19}$

Dual cements are both chemically and photo-activated to provide monomer conversion. ${ }^{20}$ However, the chemical compounds may lead to shade instability due to oxidation of the aromatic tertiary amines. However, oxidation is required to initiate polymerization. Therefore, only the use of the base pastecontaining photoinitiators-may increase stability. ${ }^{18}$ Furthermore, analysis of the initial shade stability of resin cements after cementing the dental ceramic to the tooth in the first days after the material sets must be done appropriately from a clinical point of view, since it is in these first days that the cement is subject to greater water sorption and shade change. ${ }^{20-23}$
Therefore, the aims of the present in vitro study were to evaluate:

1. the in vitro shade agreement between resin cements and their respective try-in pastes;

2. three resin cements exposed to attenuated light from a curing unit, due to the presence of a nanofluorapatite vitreous ceramic disc between the curing unit light guide and the resin cement layer; and

3. the initial shade stability of resin cements of the same simulated clinical condition, using a digital spectrophotometer.

\section{Methodology}

A total of 30 discs of microhybrid composite resin (Lliss, FGM, Joinvile, Brazil) in shade A2 were fabricated to simulate dentin, using a silicon matrix (12 $\mathrm{mm}$ in diameter $\times 5 \mathrm{~mm}$ thick). The resin was inserted in two 1.5 -mm layers, and the last $2.0-\mathrm{mm}$ thick layer was lightly pressed with a matrix polyester strip. The resin composite was light activated for 40 seconds with a LED light curing unit (Flashlite 1401, Discus Dental, Culver City, USA), with a mean light intensity of $1400 \mathrm{~mW} / \mathrm{cm}^{2}$.

Each resin specimen was manually abraded in circular motions with 220-, 360-, 400-, and 1200grit aluminum oxide abrasive paper (3M, Sumaré, Brazil). The final thickness was evaluated with a metal thickness meter (Wilcos, Sialkot, Pakistan). The specimens were washed in an ultrasonic bath (Sercon, Mogi das Cruzes, Brazil) and were stored in distilled water at $37^{\circ} \mathrm{C}$ for 24 hours.

An A2-shade nanofluorapatite vitreous ceramic disc (E-max Ceram, Ivoclar Vivadent, AG, Schaan, Germany) was used to fabricate 30 specimens. A stainless steel matrix $(12 \mathrm{~mm}$ in diameter $\times 0.5 \mathrm{~mm}$ thick) was used. The ceramic disc thickness was confirmed with a metal thickness meter (Wilcos, Sialkot, Pakistan), and the discs were polished with standardized rubber discs (Exacerapol, Edenta, Au, Switzerland), numbers 301, 306, and 322, for 30 seconds each. The polishing was performed in circular motions from the center to the periphery of the ceramic disc. After the ceramic discs were polished, they were washed in an ultrasonic bath (Sercon, Mogi das Cruzes, Brazil) and stored in distilled wa- 
ter at $24^{\circ} \mathrm{C}$ for 24 hours.

The resin composite and ceramic discs were evaluated by a previously calibrated digital spectrophotometer (EspectroShade Micro, MHT, Arbizzano di Negrar, RV, Italy) to assess the color, which was then classified according to the Vita Classical Shade Guide (Vita Zahnfabrik H. Rauter GmbH \& Co, Bad Säckingen, Germany). The resin composite blocks and ceramic discs were randomly divided into three experimental groups $(\mathrm{n}=10)$ to evaluate the colors of the try-in pastes and resin cements:

a. VIT: try-in paste Vitique shade A2 $1 / 2$ and resin cement (base paste) Vitique shade A2 $1 / 2$

b. VAR: try-in paste Variolink II shade A1 and resin cement (base paste) Variolink II shade A1

c. CHO: try-in paste Choice 2 shade A2 and resin cement (base paste) Choice 2 shade A2

The composition of the materials used in the study is presented in Table 1 .

The shade of the specimens with try-in pastes was evaluated by placing the resin composite disc on a gray-colored metal surface and applying a thin layer of paste from the respective group to the specimen. The ceramic disc was placed on the paste and pressed lightly for 5 seconds with white rubber (Faber Castell, São Paulo, Brazil) in the center of the disc to create a thin layer of try-in paste. The excess was removed with a spatula and the resin composite disc/try-in paste/ceramic disc set was submitted to shade analysis by a calibrated digital spectrophotometer.

After this procedure, the ceramic disc was removed and both ceramic and resin composite surfaces were washed with an air/water spray for 15 seconds, and cleaned with cotton wool soaked with a $99.5 \%$ acetone solution for 15 seconds.

The shade of the ceramic restoration with a resin cement layer was evaluated by cementation after the resin disc surface was etched with $37 \%$ phosphoric acid (Etch, Schaan Liechtenstein, Ivoclar Vivadent) for 10 seconds, washed with an air/water jet for 15 seconds, coated with an adhesive system (Excite) for 10 seconds, and light activated for 10 seconds. The ceramic disc was etched with $10 \%$ hydrofluoric acid for 20 seconds, washed with an air/water spray for 10 seconds, and coated with silane (Ivoclar Vivadent, Ellwangen, Germany) applied for 60 seconds. A thin coat of resin cement from the respective group was applied to the resin composite disc. The ceramic disc was placed on the resin cement and lightly pressed for 5 seconds with white rubber in the center of the disc, to create a thin resin cement layer. The excess was removed with a spatula, and

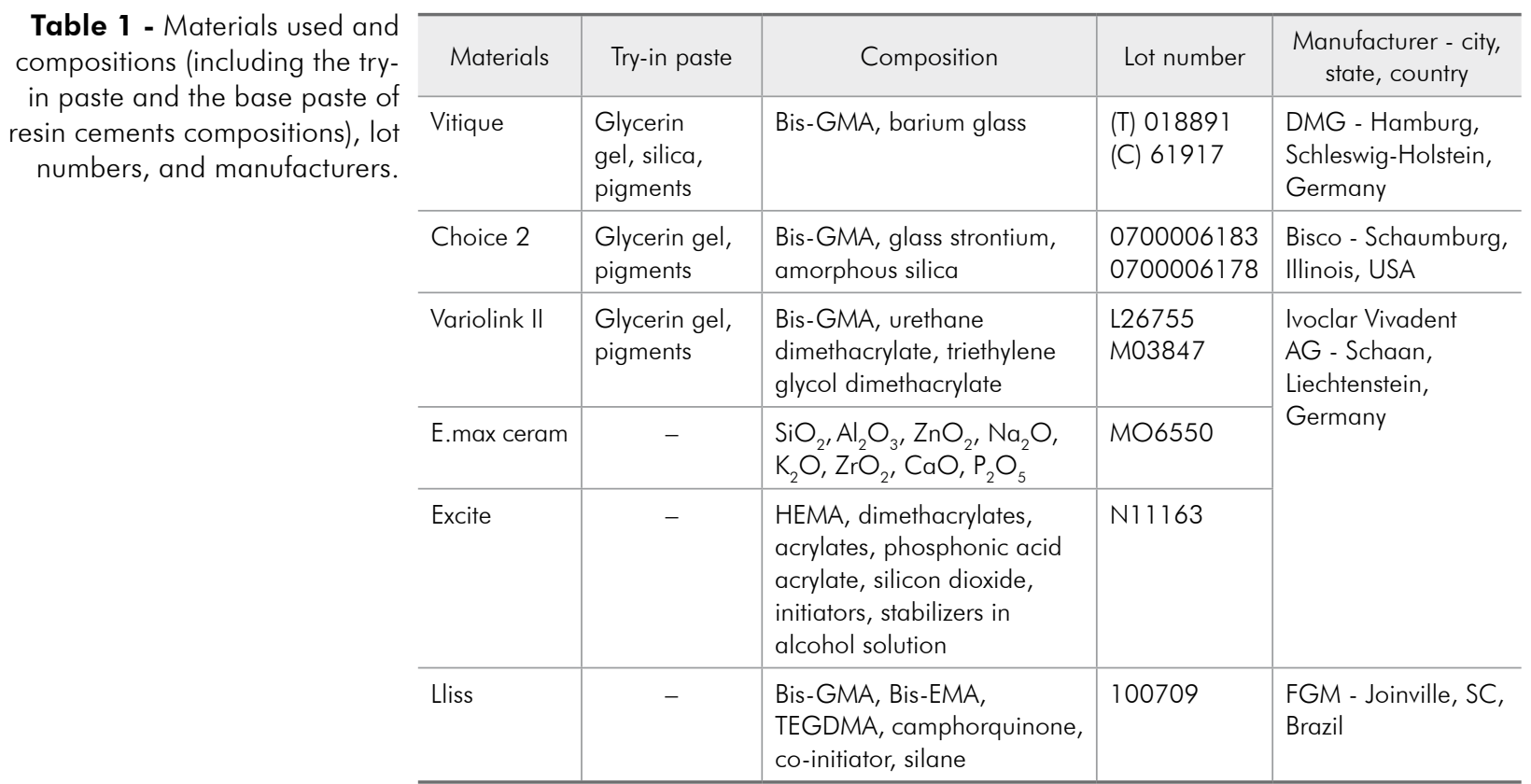



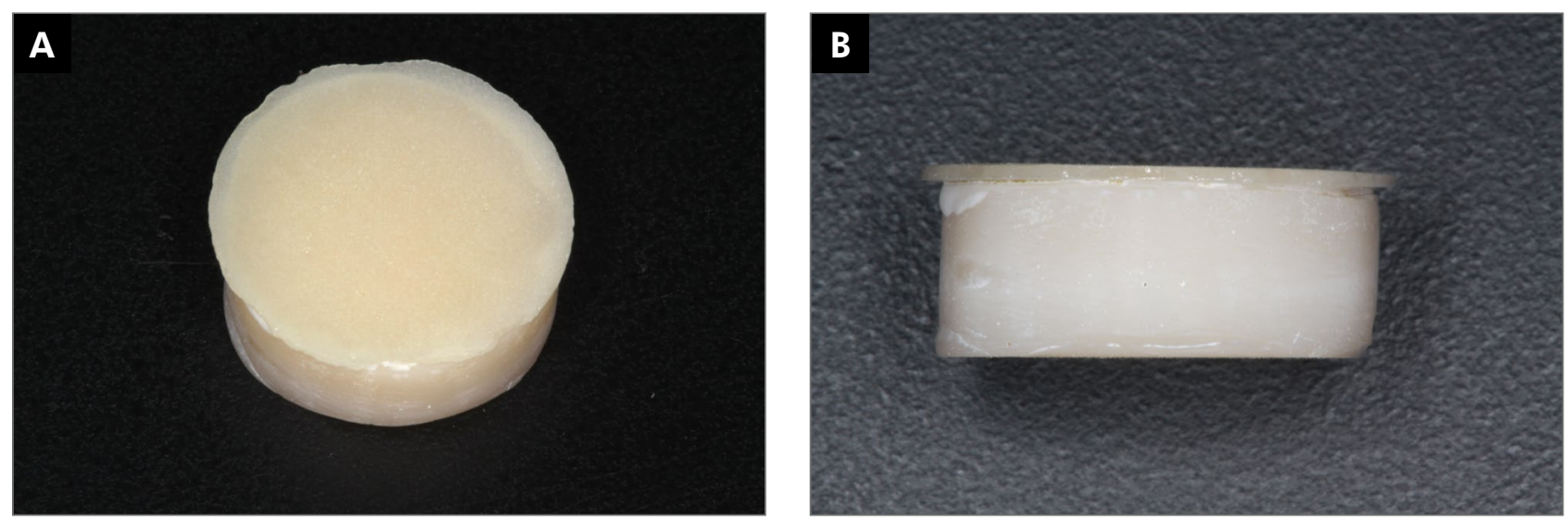

Figure 1 - Cemented test specimen. A: Top view; B: side view of the resin composite, cement and ceramic disc.

a visual examination was performed after each cementation to ensure that all specimens had resin cement layers with similar thickness (Figure 1). Light activation was performed for 40 seconds with the tip of the LED light curing unit positioned perpendicular to the ceramic disc surface.

The resin disc/resin cement/ceramic disc set was submitted to shade analysis by digital spectrophotometer, and the values of each specimen were based on an average of 5 readings. The specimens were stored in distilled water and submitted to shade analysis 2, 5, and 6 days after cementation.

The statistical analysis was obtained by performing a descriptive analysis of the data using absolute and relative frequency distribution tables. The Friedman test was applied for comparisons between intervals, and the Kruskal-Wallis and Dunn's tests, for intergroup comparisons $(\alpha=5 \%)$. Analyses were performed in Excel 2007 (Microsoft, Redmond, USA) and Bioestat 4.0 programs (Bioestat 4.0 statistical program, Mamirauá Maintainable Development Institute, Belém, Brazil).

\section{Results}

The digital spectrophotometer indicated that the shade of specimens with try-in pastes applied to the ceramic discs for the different commercial brands was A2 in $100 \%$ of the specimens, showing that two of the try-in pastes (VIT and CHO) presented shade compatibility (A2) with those recommended by the manufacturers. Immediately after cementation or at different intervals, there was no similarity in shade between the ceramic discs associated with try-in pastes and those cemented with the respective resin cements, insofar as the shade for resin cements was evaluated as predominantly A3 for all the resin cements. There was no significant difference in specimen shade between the intervals for VAR ( $\mathrm{p}=0.9709)$ and for VIT ( $\mathrm{p}=0.7458)$, according to the Friedman test. It was not possible to apply statistical analysis between the intervals for $\mathrm{CHO}$, because there was no variability between the results (Table 2).

Table 2 also shows that immediately after cementation and at Day 2, there was no significant difference in specimen shade among the cements, based on Dunn's test $(p>0.05)$. However, there was an increase in tone value towards a darker shade for the cements and in comparison with the try-in pastes, immediately after cementation and at Day 2. At Day 5 and 6 , there was no significant difference $(p=0.1260)$. At Day 6, 80\% of the specimens with VAR presented shade A3, and $20 \%$ presented shade $A 3 \frac{1}{2}$, indicating an increase in chrome when compared with immediately after cementation and at Day 2. CHO remained with no shade variation in any of the intervals. VIT presented a variation in shade in all the days, and, at Day $6,90 \%$ of the specimens showed shade A3, and $10 \%$ exhibited shade A1.

\section{Discussion}

In this study, all the try-in pastes applied to the ceramic discs presented shade similarity (A2) ac- 
Table 2 - Frequency (\%) of the results of color evaluation as a function of time for the ceramic discs with the try-in pastes or cemented with Variolink II, Choice 2, and Vitique.

\begin{tabular}{|c|c|c|c|c|c|c|}
\hline \multirow{2}{*}{$\begin{array}{l}\text { Try-in or } \\
\text { resin cement }\end{array}$} & \multirow{2}{*}{ Shade } & \multirow{2}{*}{ Try-in } & \multicolumn{4}{|c|}{ Resin cement } \\
\hline & & & Immediate & Day 2 & Day 5 & Day 6 \\
\hline \multirow{4}{*}{$\begin{array}{c}\text { VAR } \\
\text { Shade Al }\end{array}$} & $\mathrm{Al}$ & $0 \quad(0 \%)$ & $0 \quad(0 \%)$ & $0 \quad(0 \%)$ & $0 \quad(0 \%)$ & $(0 \%)$ \\
\hline & A2 & 10 (100\%) & $0 \quad(0 \%)$ & $0 \quad(0 \%)$ & $0 \quad(0 \%)$ & $0 \quad(0 \%)$ \\
\hline & A3 & $(0 \%)$ & $9 \quad(90 \%)$ & $9 \quad(90 \%)$ & $8 \quad(80 \%)$ & $8 \quad(80 \%)$ \\
\hline & $\mathrm{A} 3^{1 / 2}$ & $0 \quad(0 \%)$ & $1 \quad(10 \%)$ & $1 \quad(10 \%)$ & $2(20 \%)$ & 2 (20\%) \\
\hline \multirow{4}{*}{$\begin{array}{c}\mathrm{CHO} \\
\text { Shade A2 }\end{array}$} & $\mathrm{Al}$ & $0 \quad(0 \%)$ & $0 \quad(0 \%)$ & $0 \quad(0 \%)$ & $0 \quad(0 \%)$ & $0 \quad(0 \%)$ \\
\hline & A2 & 10 (100\%) & $0 \quad(0 \%)$ & $0 \quad(0 \%)$ & $0 \quad(0 \%)$ & $0 \quad(0 \%)$ \\
\hline & A3 & $0 \quad(0 \%)$ & 10 (100\%) & $10(100 \%)$ & $10(100 \%)$ & 10 (100\%) \\
\hline & $\mathrm{A} 3^{1 / 2}$ & $(0 \%)$ & $(0 \%)$ & $(0 \%)$ & $(0 \%)$ & $(0 \%)$ \\
\hline \multirow{4}{*}{$\begin{array}{c}\text { VIT } \\
\text { Shade A2 1/2 }\end{array}$} & $\mathrm{Al}$ & $0 \quad(0 \%)$ & $0 \quad(0 \%)$ & $0 \quad(0 \%)$ & $0 \quad(0 \%)$ & $1 \quad(10 \%)$ \\
\hline & $\mathrm{A} 2$ & 10 (100\%) & $3 \quad(30 \%)$ & $0 \quad(0 \%)$ & $0 \quad(0 \%)$ & $0 \quad(0 \%)$ \\
\hline & A3 & $(0 \%)$ & $7 \quad(70 \%)$ & $10(100 \%)$ & $10(100 \%)$ & 9 (90\%) \\
\hline & $\mathrm{A} 3^{1 / 2}$ & $(0 \%)$ & $(0 \%)$ & $(0 \%)$ & $(0 \%)$ & $(0 \%)$ \\
\hline
\end{tabular}

cording to the manufacturers' recommendations for the commercial brands evaluated. However, with regard to the cements, the ceramic discs cemented with VAR, CHO, and VIT, presented shade A3 with higher frequency, without significant difference among the intervals. The cementation performed with VIT presented shade A2 in only $30 \%$ of the specimens in the first day. The differences in shade of the cemented ceramic discs may have occurred due to the combination of the different materials (resin composite substrate + cement/ceramic disc), which may lead to a final shade differing from the shade of each material separately. It should be borne in mind that, because only the base paste of the cement was used, the polymerization was initiated by light activation alone. We must also keep in mind that when base and catalyst pastes are mixed, the cement darkens. ${ }^{1,22}$ For these reasons, the final shade of the cement may have differed from that recommended by the manufacturers, thereby influencing the results.

According to the regulations of the American Society for Testing and Materials of 1979, the ideal condition to select a shade correctly is daylight in a natural environment; however, a satisfactory shade selection can be obtained in a closed room with adequate artificial lighting and neutral-gray walls. Accordingly, the spectrophotometer-assisted shade readings were made with the specimens un- der artificial light and against a neutral-gray background. One of the important factors for cement shade stability and longevity of the restoration is knowing its physical/mechanical properties, such as composition, manipulation, and polymerization time. ${ }^{24}$ Shade change or margin staining are expected consequences resulting from the oxidation of the aromatic tertiary amine, required for initiating polymerization in chemically or dual-cured resin cements, or from inadequate polymerization. Hence, the long-term esthetics of the ceramic restoration is compromised. . $^{19,21,25}$

In the current study, $100 \%$ of the ceramic discs cemented with resin cement $\mathrm{CHO}$ presented shade stability (A3) at all intervals, whereas all the other cements promoted few and not statistically significant changes in shade over time. A factor to be considered in this study was the short time during which the specimens were stored in distilled water, as well as the short intervals between shade readings. ${ }^{18}$

Inadequate polymerization or cement type selection are factors that may cause the difference between the selected shade and the final shade obtained after the cementing procedure followed by polymerization. ${ }^{20}$ Resin cements with polymerization activated by visible light (physical) have aromatic ketones (camphorquinone) that act as photoinitiators that initiate the polymerization. 
In chemically activated cements, the initiators are tertiary amines that are activated by benzoyl peroxide, and the polymerization reaction occurs even in regions where the light from a light curing unit cannot reach the resin cement layer. For this reason, these products are indicated when thick restorations are used. However, oxidation of the amines effects shade changes of the cement over time, and allows greater marginal leakage to occur., ${ }^{719}$ Since dual cure cement effects dual activation of the setting reaction (physical and chemical), this type of cement must be chosen for higher monomer conversion to be achieved, when color stability is desired and light cannot reach deeper regions. Nevertheless, in clinical situations involving the cementation of ceramic laminate veneers, such as that performed in the present study, the ceramic laminate is thin enough to allow the light to pass through the indirect restoration. Thus, dual resin cement base paste should be selected to ensure greater color stability and control the setting time, insofar as thin laminate veneers may optimize the polymerization process and avoid the staining of the cement over time. ${ }^{5}$

Although the ceramic laminates used in the present study were only $0.5 \mathrm{~mm}$ thick, they are clinically

\section{References}

1. Sproull RC. Color matching in dentistry. Part II: Practical application of the organization of color. J Prosthet Dent. 1973 May;29(5):556-66.

2. Watson JF, Crispin BJ. Margin placement of esthetic veneer crowns. Part III: Attitudes of patients and dentists. J Prosthet Dent. 1981 May;45(5):499-501.

3. Land MF, Hopp CD. Survival rates of all-ceramic systems differ by clinical indication and fabrication method. J Evid Based Dent Pract. 2010 Mar;10(1):37-8.

4. Hamlett K. The art of veneer cementation. Alpha Omegan. 2009 Dec;102(4):128-32.

5. Burke FJ, Lucarotti OS. Ten-year outcome of porcelain laminate veneers placed within the general dental services in England and Wales. J Dent. 2009 Jan;37(1):31-8.

6. Beier US, Kapferer I, Burtscher D, Dumfahrt H. Clinical performance of porcelain laminate veneers for up to 20 years. Int J Prosthodont. 2012 Jan-Feb;25(1):79-85.

7. Noie F, O'Keefe KL, Powers JM. Color stability of resin cements after accelerated aging. Int J Prosthodont. 1995 JanFeb;8(1):51-5. favorable, due to their esthetics and function. ${ }^{6,14}$ Moreover, they were not completely translucent. When these laminates were placed on either the tryin pastes or the resin cements for shade analysis by a digital spectrophotometer, a change in shade was observed. This did not occur in the studies that used completely transparent laminate veneers. ${ }^{10,16}$

Bear in mind that try-in paste is not recommended as a method for choosing cement color. In the present study, for example, there was no similarity in shade between the try-in and its respective resin cement. Therefore, the use of try-in pastes does not represent a reliable criterion for shade selection.

\section{Conclusion}

Only two brands of try-in pastes evaluated and applied to the nanofluorapatite-based dental ceramic showed color compatibility with the try-in pastes recommended by the manufacturers. There was no similarity of shades between the ceramic discs associated with the try-in pastes and those cemented with the respective resin cements. However, there was color stability of the ceramic discs cemented with resin cements, within the evaluated intervals.

8. Aboushelib MN, Dozic A, Liem JK. Influence of framework color and layering technique on the final color of zirconia veneered restorations. Quintessence Int. 2010 May;41(5):84-9.

9. Zhang XY, Qian HX, Yang DL, Gan H, Min M, Yu WQ. [The influence of try-in pastes on the colour of all-ceramic veneers]. Shanghai Kou Qiang Yi Xue. 2007 Apr;16(2):131-5. Chinese.

10. Vichi A, Ferrari M, Davidson CL. Influence of ceramic and cement thickness on the masking of various types of opaque posts. J Prosthet Dent. 2000 Apr;83(4):412-7.

11. Xing W, Jiang T, Ma X, Liang S, Wang Z, Sa Y, et al. Evaluation of the esthetic effect of resin cements and try-in pastes on ceromer veneers. J Dent. 2010 May;38(7):87-94.

12. Prata RA, Oliveira VP, Menezes FC, Borges GA, Andrade OS, Gonçalves LS. Effect of 'Try-in' paste removal method on bond strength to lithium disilicate ceramic. J Dent. 2011 Dec;39(12):863-70.

13. Uludag B, Ozturk O, Ozturk AN. Microleakage of ceramic inlays luted with different resin cements and dentin adhesives. J Prosthet Dent. 2009 Oct;102(4):235-41. 
Color agreement between nanofluorapatite ceramic discs associated with try-in pastes and with resin cements

14. Balderamos LP, O’Keefe KL, Powers JM. Color accuracy of resin cements and try-in pastes. Int J Prosthodont. 1997 MarApr;10(2):111-5.

15. Alghazali N, Laukner J, Burnside G, Jarad FD, Smith PW, Preston AJ. An investigation into the effect of try-in pastes, uncured and cured resin cements on the overall color of ceramic veneer restorations: an in vitro study. J Dent. 2010; 38 Suppl 2:e78-86.

16. Brauer GM. Color changes of composites on exposure to various energy sources. Dent Mater. 1988 Apr;4(2):55-9.

17. Inokoshi S, Burrow MF, Kataumi M, Yamada T, Takatsu T. Opacity and color changes of tooth-colored restorative materials. Oper Dent. 1996 Mar-Apr;21(2):73-80.

18. Nathanson D, Banasr F. Color stability of resin cement an in vitro study. Pract Proced Aesthet Dent. 2002 Aug;14(6):44955.

19. Smith DS, Vandewalle KS, Whisler G. Color stability of composite resin cements. Gen Dent. 2011 Sep-Oct;59(5):390-4.
20. Seghi RR, Johnston WM, O’Brien WJ. Performance assessment of colorimetric devices on dental porcelains. J Dent Res. 1989;68(12):1755-9.

21. Clark EB. The color problem in dentistry. Dent Dig. 1931;37:571-82.

22. Peters AD, Meiers JC. Effect of polymerization mode of a dual cured resin cement on time-dependent shear bond strength to porcelain. Am J Dent. 1996 Dec;9(6):264-8.

23. Berrong JM, Weed RM, Schwartz IS. Color stability of select dual-cure composite resin cements. J Prosthodont. 1993 Mar;2(1):24-7.

24. Kious AR, Roberts HW, Brakett WW. Film thickness of recently introduced luting cements. J Prosthet Dent. 2009 Mar;101(3):189-92.

25. Barghi N, Chung K, Farshian F, Berry T. Effects of the solvents on bond strength of resin bonded porcelain. J Oral Rehabil. 1999 Nov;26(11):853-7. 